Max-Planck-Institut für demografische Forschung

Max Planck Institute for Demographic Research

Konrad-Zuse-Strasse 1 - D-18057 Rostock · GERMANY

Tel +49 (0) 3812081 - 0; Fax +49 (0) 3812081 - 202;

http://www.demogr.mpg.de

MPIDR WORKING PAPER WP 2010-019

FEBRUARY 2010

\title{
Adaptive trade-off in C. capitata is a characteristic feature of the long-lived subpopulation
}

Alexey A. Romanyukha (eburg@inm.ras.ru)

Arseniy S. Karkach (arseny@mail.ru)

James Carey (jrcarey@ucdavis.edu)

Anatoli I. Yashin (aiy@duke.edu)

This working paper has been approved for release by: James W. Vaupel (jwv@demogr.mpg.de), Head of the Laboratory of Survival and Longevity.

(C) Copyright is held by the authors.

Working papers of the Max Planck Institute for Demographic Research receive only limited review. Views or opinions expressed in working papers are attributable to the authors and do not necessarily reflect those of the Institute. 


\title{
Adaptive trade-off in C. capitata is a characteristic feature of the long-lived subpopulation
}

\author{
MPIDR working paper
}

August 2009

\begin{abstract}
Alexey A. Romanyukha ${ }^{1}$, Arseniy S. Karkach ${ }^{1}$, James Carey ${ }^{2}$, Anatoli I. Yashin ${ }^{3}$
${ }^{1}$ Institute of Numerical Mathematics, Russian Academy of Sciences, Gubkina 8, 119333, Moscow, Russia. Phone +7 495 9383765; Email: eburg@,inm.ras.ru; +7 495 9383990; Email: arseny@mail.ru

${ }^{2}$ University of California at Davis, Department of Entomology, 67 Briggs Hall, CA, USA Phone: (530) 752-6217; Email: jrcarey@ucdavis.edu

${ }^{3}$ Duke University, Center for Demographic Studies, 2117 Campus Drive, Box 90408, Durham, NC 27708-0408, USA Phone (919) 668 2713; email: aiy@duke.edu
\end{abstract}

\begin{abstract}
The conception of the cost of reproduction provides an important insight on connection between fertility and life span in living organisms. Despite substantial progress in understanding this connection many important features of fertility-longevity trade-off are masked by confounding factors, and remain poorly understood. We performed reanalysis of experimental data on fertility and longevity in medflies and discovered a physiological fertility-longevity trade-off especially pronounced in the long lived fraction of the population. The presence of such trade-off suggests existence of common metabolic resource shared between reproduction and somatic maintenance in a fly. We show that in addition to sugar, protein and any other essential components of the yeast the presence of at least one more component in the diet is required to explain the adaptive trade-off between longevity and reproduction in response to environmental conditions. We suggest and discuss a principle of dynamic resource allocation which explains the fertility-longevity trade-off phenomenon for these flies. Adaptive allocation of metabolic and other resources allows flies to tailor their life history parameters to the environment. Due to limitations of the physiological adaptation different individuals may be genetically "preadapted" to different living conditions thus contributing to population stability and heterogeneity.
\end{abstract}

Keywords: adaptation, life history, cost of reproduction, coordinated aging, fitness 


\section{Introduction}

The rate of aging is affected by various processes of physiological adaptation (Bitterman et al, 2003). Aging is related to molecular repair and turnover and homeostasis maintenance which are costly processes accounting for significant proportion of TEE (Rolfe and Brown, 1997). Physiological processes compete for resources, e.g. reproduction affects the energy budget related to maintenance of homeostasis and cell repair thus changing the rate of aging and longevity. This poses two questions: what is the nature of shared resources, and under what conditions their allocation alters the rate of ageing? Of particular interest is studying this phenomenon in conditions similar to those experienced by species in nature.

It was suggested that a significant limitation of food quantity or quality may be needed to observe a trade-off between longevity and fertility (Stearns, 1992). The effects of dietary restriction (DR) on fertility and longevity have been investigated in Drosophila and C. capitata across a large range of treatments (Graves, 1993; Chapman and Partridge, 1996; Chapman et al., 1998; Good and Tatar, 2001; Piper et al., 2005). The experimental results, however, look controversial and do not explain why trade off is manifested in some cases and not in others. Experiment of Carey et al. (2002) was one of the first to include in a single study such aspects as monitoring daily survival of large numbers of both sexes subjected to multiple DR treatments across both caloric and sugar-yeast gradients, with age-specific and lifetime reproduction measured in females.

Since then several important works have emerged. Lee et al. (2008) question the lifeextending role of DR and study lifespan and reproduction in Drosophila across gradients of sugar and protein concentration in diet while measuring the actual food consumption. They studied the fly behavior in conditions when the flies could and could not choose the food composition. The "optimal" food composition was shown to exist and be selected by the flies when they have choice. Carey et al. (2008) study the conditions under which the longevityenhancing effects resulting from food restriction either counteract, complement or reinforce the mortality costs of reproduction. The sex-specific survival and daily reproduction in females of the tephritid fruit fly, Anastrepha ludens was analyzed over a fine grid of sugar- and proteinrestricted diets.

Out main objective was to study the principles and limitations of the lifetime physiological adaptation. We believe that experiment of Carey et al., (2002) has the ability to shed more light on the problem of longevity-fertility trade-off and reanalyze its' data from a different perspective taking into account the population heterogeneity in the level and distribution of resources. In this experiment cohorts of 100 individual female medflies were subjected to periodical diet treatments consisting of days of ad libitum sugar-only diet (no protein) and days of ad libitum sugar and yeast hydrolysate (protein) diet. The sugar-only diet did not support substantial reproduction but periodical inclusions of yeast hydrolysate activated egg-laying. The cohortaverage fertility and longevity demonstrate no association when the yeast hydrolysate availability changes.

The conditions and feeding were strictly controlled and flies emerged from selected pupae of the same size. Nevertheless the fly cohorts were highly heterogeneous in longevity and fertility the principal characteristics of fitness. It is generally noted that the population heterogeneity complicates detection of fertility-longevity trade-offs (Arking, 1998; Zera and Harshman, 2001). This motivated us to compare longevity and fertility on different diets separately for the longlived and short-lived flies. We discovered a strong negative association between these characteristics in the long-lived, but not in the short-lived flies. Namely, in the long-lived flies increasing frequency of complete adult diet days led to decreasing of life expectancy and increasing of average total fertility rate.

To describe this association we suggest a two-resource model of adaptive regulation of individual maintenance and reproduction. The work builds upon a previous attempt of mechanistic modeling of processes related to feeding, lifespan and fertility in Medfly females 
(Romanyukha et al., 2004).

\section{Materials and methods}

Pairs of Mediterranean fruit fly (C. capitata) females and males were housed in plastic containers and subjected to different dietary regimes composed of two diets: the "complete adult" diet containing yeast hydrolysate, sugar, water and vitamins (diet $p$ ) and a "sugar-only" diet (diet s) containing pure sucrose and water (Carey et al., 2002). Female life span and the number of eggs laid daily were studied in several cohorts subject to different cyclic dietary treatments. Five cohorts received food in cycles of one day diet $p$ and 1, 3, 5, 10, and 20 days diet $s$. One control cohort received diet $s$ full time and one received diet $p$ full time. Following Carey et al. (2002) we denote the cohorts which received cycle diets as 1:1, 1:3, 1:5, 1:10, and 1:20. The controls will be referred to as $\mathbf{p}$ and $\mathbf{s}$. Each cohort contained 100 females initially. Special attention has been paid to population homogeneity. For this purpose pupae were selected close in size, fly couples were maintained in identical containers and received standardized diet.

\section{Results}

Analysis of data reveals high variance of life span and especially total number of eggs laid in all cohorts (Table 1, columns 2 and 3). We wanted to understand whether the changes in diet affect observed life history traits and how. High data variance suggested choosing more homogenous groups. In each dietary cohort we selected $25 \%$ flies with the highest life span (the "long-lived flies"), and $25 \%$ with the lowest life span (the "short-lived flies") and calculated life expectancy $\left(\mathrm{e}_{0}\right)$ and Total Fertility Rate (TFR) in selected groups and whole cohorts (Table 1).

Table 1. Life expectancy ( $\mathrm{e}_{0}$, days) and total fertility rate (TFR, eggs) of female medflies subject to 7 treatments; $\mathrm{e}_{0}=$ expectation of life at eclosion (days); SD = standard deviation; number of flies in groups given in brackets

\begin{tabular}{c|cc|cc|cc}
\hline & \multicolumn{2}{|c|}{ All flies (100) } & \multicolumn{2}{c|}{ Short lived (25) } & \multicolumn{2}{c}{ Long lived (25) } \\
\hline \multirow{4}{*}{ Diet } & $\mathrm{e}_{0}$ & $\mathrm{TFR}$ & $\mathrm{e}_{0}$ & TFR & $\mathrm{e}_{0}$ & $\bar{F}$ \\
\hline $\mathbf{S}$ & $\mathrm{SD}$ & $\mathrm{SD}$ & $\mathrm{SD}$ & $\mathrm{SD}$ & $\mathrm{SD}$ & $\mathrm{SD}$ \\
\hline $\mathbf{p}$ & 44 & 52 & 12 & 29 & 80 & 74 \\
& 27 & 50 & 5 & 40 & 9 & 55 \\
$\mathbf{1 : 1}$ & 40 & 888 & 21 & 299 & 56 & 1034 \\
& 14 & 490 & 8 & 265 & 8 & 445 \\
$\mathbf{1 : 3}$ & 37 & 709 & 13 & 121 & 58 & 997 \\
& 18 & 500 & 6 & 130 & 8 & 450 \\
$\mathbf{1 : 5}$ & 43 & 490 & 14 & 116 & 74 & 679 \\
& 24 & 390 & 6 & 150 & 19 & 470 \\
$\mathbf{1 : 1 0}$ & 47 & 458 & 14 & 84 & 79 & 708 \\
& 25 & 320 & 7 & 445 & 15 & 255 \\
$\mathbf{1 : 2 0}$ & 50 & 296 & 12 & 93 & 86 & 438 \\
& 29 & 220 & 5 & 360 & 14 & 260 \\
& 48 & 174 & 11 & 95 & 93 & 267 \\
\hline
\end{tabular}

The "short lived" are $25 \%$ flies with the lowest life spans, and the "long lived" are $25 \%$ flies with the highest life spans in each cohort.

Fig. 1 displays values of $\mathrm{e}_{0}$ and TFR for entire cohorts and groups of short- and long-lived flies. The negative relation between average fertility and life span in the long-lived flies can be approximated by a regression line:

$0.05 \mathrm{TFR}+\mathrm{e}_{0}=109$ 
more days with diet $p$ lead to higher fertility and shorter life span. For flies in short-lived groups all diets result in closer and relatively small values of $\mathrm{e}_{0}$ and TFR. For example life expectancy of long-lived flies on diet 1:20 is 37 days higher than on diet $\mathbf{p}$ and expectancy on diet 1:1 is close to that on diet $\mathbf{p}$. However the life expectancy in cohort 1:20 is 13 days higher that in the cohort $\mathbf{s}$, the flies also laid 3.6 times more eggs. Hence the long-lived flies on diet 1:20 which received complete diet only 3 or 4 times in life did not only achieve significant reproduction but also survived much longer than those on sugar-only diet.

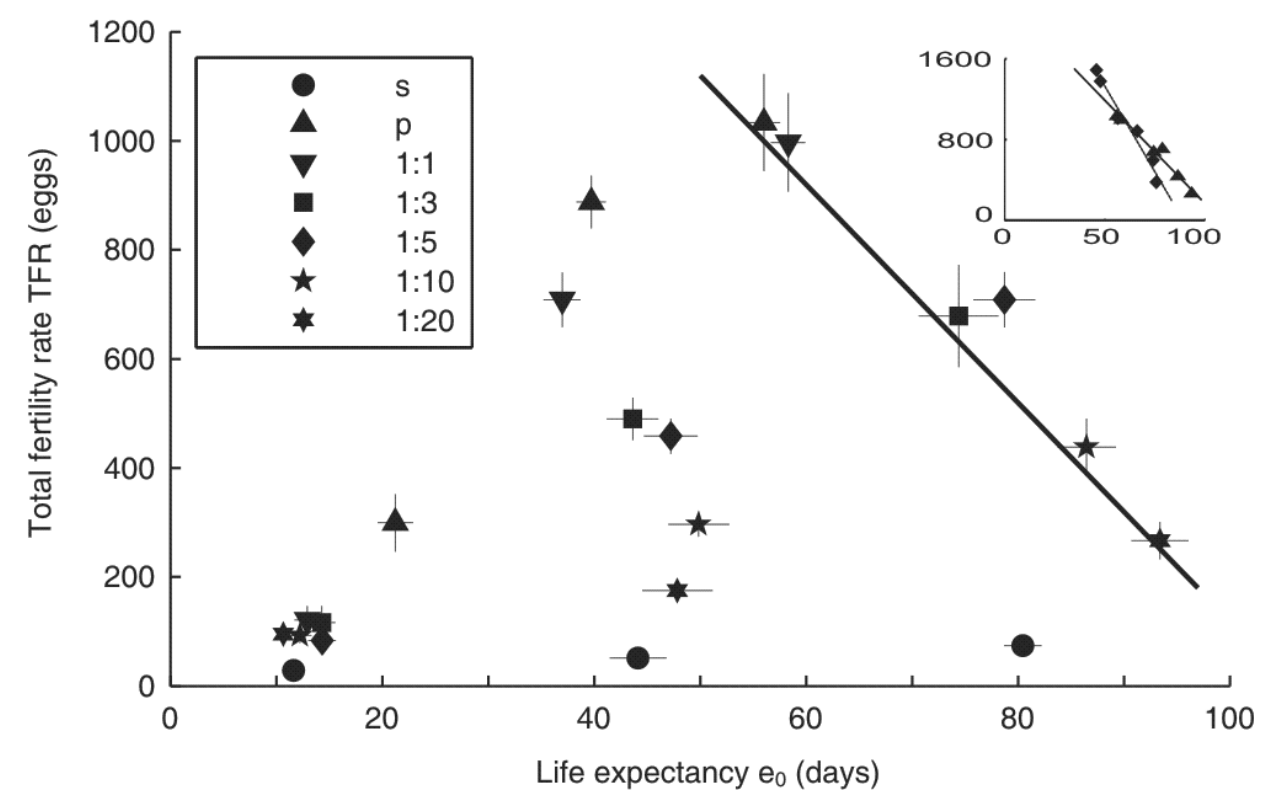

Fig. 1. Life expectancy $\left(\mathrm{e}_{0}\right)$ and average total fertility rate (TFR) for flies subject to different treatments in groups of 25\% short-lived (left), all flies (middle) and $25 \%$ long-lived (right). The regression line is fitted to the averages in the long-lived groups (group $s$ excluded). The whiskers show standard error of the mean. Inset: comparison of regression lines for $25 \%$ long-lived (triangles, line) and 25\% most fertile flies (diamonds, broken line).

Linear relationships similar to Fig. 1 were observed when selecting groups of $10 \%$ to $30 \%$ flies with longest life spans from each cohort. To check whether a similar relation exists in flies selected by fertility we selected in a similar way $25 \%$ flies with lowest, and $25 \%$ flies with highest total fertility from each cohort. Average life spans and fertilities in groups of $25 \%$ "most reproductive flies" and a corresponding regression line are shown on the inset to Fig. 1. The groups of most fertile flies show a negative relation between life span and fertility as well (on average there are $70 \%$ common flies between groups of $25 \%$ most long-lived and $25 \%$ most fertile flies in all cohorts). Corresponding regression line is steeper and average fertilities are higher compared to selection by life spans. Groups with lowest $(25 \%)$ and intermediate $(50 \%)$ reproduction have characteristics close to groups selected by life span (data not shown).

\section{Discussion}

Trade-off and allocation. Negative associations of life history traits with each other are generally referred to as life history trade-offs (Reznick 1985). Accordingly we can regard the relation between average lifetime reproduction and life expectancy manifested in long-lived and highly reproductive medfly females (Fig. 1) as a physiological trade-off (Stearns 1992).

The essence of the trade-off phenomenon is allocation of limited resource(s). The duration of life and fertility are affected by resources allocated to corresponding functions (Kirkwood and Shanley, 2005) hence the linear relation (1) can be interpreted as follows. Suppose that some 
hypothetical resource $(R)$ is shared between somatic maintenance and reproduction. Further assume that lifespan is proportional to resource spent on somatic maintenance and the total amount of eggs laid is proportional to amount of resource spent on reproduction; the resource can be reallocated between maintenance and reproduction and is used up completely by the end of life. This allows translating observed life history characteristics (lifespan, fertility rate) into unobserved physiological (amount of resources). Denoting the amount of resource allocated to reproduction by $R_{f}=0.05 \mathrm{TFR}$, and amount allocated to somatic maintenance by $R_{m}=\mathrm{e}_{0}$, we obtain that $R_{f}+R_{m}=R$. Note that on all diets the average initial values of resource (at eclosion) in the long-lived flies are close to $\bar{R}=109$ (U, arbitrary units, measured here in days of life). For example, in experiment 1:10 $R_{m}=86, R_{f}=21.9$ hence resources on average were allocated in proportion 20:80. On other diets the proportion of allocation was different. The group $\mathbf{s}$ is an outlier supposedly because the female flies could not reproduce and therefore observed $R_{f}$ was close to zero (see Fig. 1).

Adaptive nature of trade-off. The ability to live longer or to achieve higher reproduction at the expense of longevity may be a useful adaptive feature helping to adapt to unpredictable environmental conditions. It allows female flies to survive the periods of protein shortage when the reproduction is limited and to increase fitness by achieving additional lifespan. We call the phenomenon seen in Fig. 1 and expressed in Eqn. 1 an adaptive trade-off.

Coordinated aging. Physiological adaptation to environment involves metabolic regulation and uses environmental cues to predict the changes (Simmons and Bradley, 1997; Harshman and Zera, 2007). The adaptation may involve changing levels of somatic repair, homeostasis maintenance and reproduction. Some of these processes may share common resources (replaceable, partially or non-replaceable) and hence compete.

The total lifetime reproduction, $F$, is determined by daily reproduction and life span. The daily reproduction is limited by metabolic capacity of the reproductive system and availability of essential substrates in food. When the yeast availability is poor the daily reproduction is low and in this case the long life is advantageous since it allows increasing total reproduction. In this case the maximum total reproduction is achieved by flies utilizing all available substrate (and metabolic resource as required) for egg production and using the remaining metabolic resource for somatic maintenance and life support. Such strategy results in total metabolic resource $R$ being almost used up by the end of life and highest total reproduction possible for the diet.

The data shown in Fig. 1 allow estimating the relative cost of reproduction and maintenance (Reznick, 1985; Partridge and Gems, 2006). Eqn. 1 describes total fertility rate and life expectancy in the long-lived flies. The same initial amount of metabolic resource may be allocated so that the total fertility (or fitness) is maximized. Such redistribution of metabolic resource between processes of maintenance and reproduction results in the adaptive trade-off between life span and reproduction. Coordinated depletion of resource allocated to reproduction and maintenance can be called coordinated aging.

"Reproductive" and "resistant" flies. Preadapted flies. It is well established that the phenotypic adaptation is limited and organisms of a certain genotype are unable to adjust and perform best on the whole spectrum of possible environmental conditions. The natural populations are genetically heterogeneous and contain a spectrum of genotypes which may be "preadapted" to different environmental conditions; this extends the range of conditions in which the population survives and raises the population stability.

Comparison of groups of long- and short-lived flies suggests that a huge difference in the average level of resource exists. $\bar{R}$ equals $109 \mathrm{U}$ in the long-lived flies and only $13-36 \mathrm{U}$ in the short-lived ones (estimated from Table 1 using relation $\bar{R}=e_{0}+0.05 T F R$ ). Such variability in the initial level of metabolic resource may reflect differences in individual fitness.

The short-lived females lay 3 to 8 times less eggs than the long-lived ones and hence have 
much lower fitness. But since such flies remain in natural populations, there must be traits which make them competitive. Survival and reproduction in different environments may benefit from traits that flies could not take advantage of in described experiments. E.g. the experimental conditions are much more stable and comfortable than those in the wild. The flies that had short life in the experiment could possibly achieve longer life and higher reproduction in other conditions such as dehydration (Hoffmann and Parsons, 1989), infections (Kraaijeveld and Godfray, 1997; Fellowes and Godfray, 2000), damage (Plaistow et al., 2003) etc. And viceversa: the flies that were successful in laboratory could have relatively lower fitness in other environments. Also the strategy of quick assimilation and metabolizing of energy and substrates which gave advantage in the experiments is advantageous only in certain environments.

Carey et al. (1998) proposed that there are two metabolic modes in which the flies can be: a waiting mode in which both mortality and reproduction are low and a reproductive mode in which mortality is very low at the onset of egg laying but accelerates as eggs are laid. Medflies stay in waiting mode when they are fed only sugar. When fed protein, a scarce resource in the wild, medflies switch to reproductive mode. We think that our idea of resource allocation and "preadaptation" of parts of population to certain conditions goes in line with this idea but extends it and explain the biological mechanism of the phenomenon.

It is important to note that allocation of resources between reproduction and repair/maintenance may have several stress-specific aspects. Changes in ambient temperature, humidity, radiation, or other environmental factors may invoke different responses. The flies with weak resistance to dietary changes may have high resistance to changes in the temperature, humidity, or other environmental factors. Such property is well established in microorganisms that increase their resistance to unfavorable environment by decreasing metabolic rate (Brown et al., 1988). Such ability to selectively and specifically respond to different stressors by using distinct properties of physiological machinery might increase fitness of the entire fly population in variable natural environment.

Individual adaptation of stress resistance and metabolism during life is also impeded by physiological limitations (Bubliy and Loeschcke, 2005) so parts of the population can be preadapted to certain unfavorable environmental conditions to achieve a higher population survival and fitness. Such subpopulations may manifest low fitness in specific conditions but perform better in other.

Biological interpretation of the shared resource. Longevity is largely determined by somatic maintenance. Both maintenance and reproduction rely on the processes of synthesis which are powered by mitochondria producing ATP (Highnam and Hill, 1977). In this relation the common metabolic resource may be the energy-producing capacity of mitochondria. Produced energy is used to maintain homeostasis, synthesize macromolecules and perform various processes. The mitochondria are located in the tissues and feed them with energy locally since ATP can not be transported long distance. Hence part of the organism mitochondrial power can be used only for reproduction or for repair and maintenance.

Mitochondria activity is related to their powering activity. ATP production is accompanied by production of oxidative radicals which damage mitochondria. This leads to gradual decay of their function, aging, and desctruction (Vain and Webster, 1977; Miguel and Fleming, 1986; Kalous and Drahota, 1996; Huss and Kelly, 2005; Schlattner et al., 2006). Hence the powering capacity of mitochondria is limited and allocation of mitochondrial power can be the physiological basis for the fertility-longevity trade-off. We will refer to this as metabolic resource $R$.

Another resource is replaceable and is obtained from the yeast hydrolysate. Compounds of the complete diet increase eggs production 17 times in cohort $\mathbf{p}$ compared with sugar-only diet (cohort s, complete cohorts compared). Such increase was attributed to greater amino acid intake (Carey et al., 2002; O'Brien et al., 2002). We think that other essential components of the yeast hydrolysate could be sterols (ergosterol and cholesterol). These compounds are used in synthesis of steroid hormones which play critical role in ontogenesis (Gilbert et al., 2002). This 
resource will be further referred to as $\Lambda$.

Analysis of more experimental data on diet influence on life expectancy and reproduction will be required to check the generality of the fertility-longevity trade-off observed in the "successful" individuals of the population.

\section{Acknowledgement}

The authors would like to thank James Vaupel and Joshua Goldstein for the opportunity of using resources of the Max Planck Institute for Demographic Research in Germany, during work on this manuscript.

\section{References}

Arking, R., 1998. Biology of aging. Observations and principles, 2nd ed. Sinauer Associates, Sunderland, Massachusetts USA.

Bitterman, K.J., Medvedik, O., Sinclair, D.A., 2003. Longevity regulation in Saccharomyces cerevisiae: linking metabolism, genome stability, and heterochromatin. Microbiology and molecular biology reviews 67:3, 376-399.

Brown, M.R., Allison, D.G., Gilbert, P., 1988. Resistance of bacterial biofilms to antibiotics a growth-rate related effect? J. Antimicrob. Chemother 22, 777-780.

Bubliy, O.A., Loeschcke, V., 2005. Correlated responses to selection for stress resistance and longevity in a laboratory population of Drosophila melanogaster. J. Evol. Biol. 18, 789-803.

Carey, J.R., Harshman, L.G., Liedo, P., Müller, H.-G., Wang, J.-L. and Zhang, Z., 2008. Longevity-fertility trade-offs in the tephritid fruit fly, Anastrepha ludens, across dietaryrestriction gradients. Aging Cell 7, 470-477.

Carey, J.R., Liedo, P., Harshman, L., Liu, X., Muller, H.-G., Partridge, L., Wang, J.-L., 2002. Food pulses increase longevity and induce cyclical egg production in Mediterranean fruit flies. Functional Ecology 16, 313-325.

Carey, J.R., Liedo, P., Muller, H.-G, Wang, J.-L., Vaupel, J.W., 1998. Dual Modes of Aging in Mediterranean Fruit Fly Females. Science 281, 996-998.

Chapman, T., Miyatake, T., Smith, H.K., Partridge, L., 1998. Interactions of mating, egg production and death rates in females of the Mediterranean fruit fly, Ceratitis capitata. Proc. Biol. Sci. 265, 1879-1894.

Chapman, T., Partridge, L., 1996. Female fitness in Drosophila melanogaster: an interaction between the effect of nutrition and of encounter rate with males. Proceedings of the Royal Society of London, Series B, 263, 755-759.

Fellowes, M.D.E., Godfray, H.C.J., 2000. The evolutionary ecology of resistance to parasitoids by Drosophila. Heredity $84,1-8$.

Gilbert, L., Rybczynski, R., Warren, J., 2002. Control and biochemical nature of the ecdysteroidogenic pathway. Annu Rev. Entomol. 47, 883-916.

Good, T.P., Tatar, M., 2001. Age-specific mortality and reproduction respond to adult dietary restriction in Drosophila melanogaster. J.Insect Physiol. 47, 1467-1473.

Graves, J.L., 1993. The costs of reproduction and dietary restriction: parallels between insects and mammals. Growth Dev. Aging. 57, 233-249.

Harshman, L.G., Zera, A.J., 2007. The cost of reproduction: the devil in the details. Trends in Ecology \& Evolution 22: 2, 80-86.

Highnam, K.C., Hill, L., 1977. The Comparative Endocrinology of the Invertebrates, 2nd ed. Arnold, London.

Hoffmann, A.A., Parsons, P.A., 1989. Selection for Increased Desiccation Resistance in Drosophila melanogaster: Additive Genetic Control and Correlated Responses for Other Stresses. Genetics 122, 837-845.

Huss, J.M., Kelly, D.P., 2005. Mitochondrial energy metabolism in heart failure: a question of balance. J. Clin. Invest 115, 547-555. 
Kalous, M., Drahota, Z., 1996. The role of mitochondria in aging. Physiol. Res. 45, 351-359.

Kirkwood, T.B.L., Shanley, D.P., 2005. Food restriction, evolution and ageing. Mech. Ageing Dev. 126, 1011-1016.

Kraaijeveld, A.R., Godfray, H.C.J., 1997. Trade-off between parasitoid resistance and larval competitive ability in Drosophila melanogaster. Nature 389, 278-280.

Lee, K.P., Simpson, S.J., Clissold, F.J., Brooks,R., Ballard, J.W.O., Taylor, P.W., Soran, N., Raubenheimer D., 2008. Lifespan and reproduction in Drosophila: New insights from nutritional geometry. PNAS 105:7, 2498-2503.

Miguel, J., Fleming, J.E., 1986. Theoretical end experimental support for an "oxygen radicalmitochondrial injury" hypothesis of cell aging. In: Johnson, J., Walford, R., Harman, D., Miquel, J. (Eds). Radicals, Aging, and Degenerative Diseases. Alan R. Liss , pp 51-74.

O’Brien, D., Fogel, M., Boggs, C., 2002. Renewable and nonrenewable resources: Amino acid turnover and allocation to reproduction in Lepidoptera. PNAS 99, 4413-4418.

Partridge, L., Gems, D., 2006. Beyond the evolutionary theory of ageing, from functional genomics to evo-gero. Trends in Ecology and Evolution 21, 334-340.

Piper, M.D.W., Mair, W., Partridge, L., 2005. Counting the calories: the role of specific nutrients in extension of life span by food restriction. J. Gerontol. A Biol. Sci. Med. Sci. 60, 549-555.

Plaistow, S.J., Outreman, Y., Moret, Y., Rigau, T., 2003. Variation in the risk of being wounded: an overlooked factor in studies of invertebrate immune function? Ecology Letters 6, 489-494.

Reznick, D., 1985. Cost of reproduction: an evaluation of the empirical evidence. Oikos 44, 257-267.

Rolfe, D.F.S., Brown, G.C., 1997. Cellular energy utilization and molecular origin of standard metabolic rate in mammals. Physiological Reviews 77:3, 731-758.

Romanyukha, A.A., Carey, J.R., Karkach, A.S., Yashin, A.I., 2004. The impact of diet switching on resource allocation to reproduction and longevity in Mediterranean fruitflies. Proc. R. Soc. Lond. B. 271, 1319-1324.

Schlattner, U., Tokarska-Schlattner, M., Wallimann, T., 2006. Mitochondrial creatine kinase in human health and disease. Biochimica et Biophysica Acta 1762, 164-180.

Stearns, S.C., 1992. The Evolution of Life Histories. Oxford : Oxford University Press.

Vain, A.C., Webster, G.C., 1977. Age-related changes in mitochondrial function in Drosophila melanogaster. Exp. Gerontol. 12, 1-5.

Zera, A.J., Harshman, L.G., 2001. The physiology of life history trade-offs in animals. Annu. Rev. Ecol. Syst. 32, 95-126. 